Archivist

\title{
Specialist retrieval teams for transfer to regional paediatric intensive care units: effect on mortality
}

In England and Wales paediatric intensive care is provided by 29 regional paediatric intensive care units (PICUs) with specialist retrieval teams to transfer critically ill children from other hospitals. Data from the Paediatric Intensive Care Audit Network on children (aged 16 years or younger) admitted to these units between 1 January 2005 and 31 December 2008 have been analysed in a retrospective cohort study to assess the effectiveness of the specialist retrieval teams (Padmanabhan Ramnarayan and colleagues. Lancet 2010;376:698-704; see also Comment, ibid: 660-1).

During these 4 years there were 57997 admissions to the 29 PICUs, of which 23530 were planned admissions and 34399 unplanned (68 admissions were unclassified). Complete data on source of admission were available for 33492 of the unplanned admissions and 17649 were transfers from other hospitals. The type of transferring team (specialist or non-specialist) was known for 16875 cases and was specialist in 13729 (81\%). Compared with children transferred to PICUs from within the same hospital, children transferred from other hospitals were younger (median age 10 months vs 18 months), more acutely ill (mortality risk 6\% vs 4\% using the Paediatric Index of Mortality), needed more resources (such as invasive ventilation, vasoactive drugs, renal replacement therapy, extracorporeal membrane oxygenation and/or multiple-organ support), had longer stays in the PICU (median 75 h vs $43 \mathrm{~h}$ ) and had a higher crude mortality ( $8 \%$ vs $6 \%$ ). On multivariable analysis after adjustment for case mix and organisational factors, the risk of death among interhospital transfers was significantly (35\%) lower than among intrahospital transfers. With similar analysis, the times spent in PICU did not differ significantly between these two groups. When the type of transferring team was considered, crude mortality was similar with specialist and non-specialist teams, although the children transferred by the specialist teams were more severely ill. On multivariable analysis, the risk of death was $42 \%$ lower with specialist team transfer. The median distance travelled (from home postcode to the PICU) for children transferred from other hospitals was $31 \mathrm{~km}$ (37 km for transfer to PICUs outside London). The risk of death was not related significantly to distance travelled and children not admitted to their nearest PICU did not have a higher risk of death. (Not being admitted to the nearest PICU added a median distance of $8 \mathrm{~km}$ to the journey in London and $20 \mathrm{~km}$ outside London.)

These findings appear to confirm the value of specialist retrieval teams. Why children transferred from other hospitals did better than children transferred to the PICU in the same hospital is not explained.

Provenance and peer review Not commissioned; not externally peer reviewed. 